\title{
El ritmo circadiano de la presión arterial y su relación con los factores de riesgo cardiovascular
}

\author{
Cristina García Serrano, Marta Micol Bachiller, David Betrán Biurrun, Lídia Aran Solé, Jesús Pujol Salud \\ Centro de Atención Primaria de Balaguer. Lleida. España
}

Como citar este artículo:

García-Serrano C, Micol-Bachiller M, Betrán-Biurrun D, Aran-Solé L, Pujol-Salud J. El ritmo circadiano de la presión arterial y su relación con los factores de riesgo cardiovascular. 2019 Abr-Jun;22(2):151-8

\section{Resumen}

Introducción: Numerosos estudios han establecido tanto la hipertensión arterial como la hipertensión de bata blanca como factores de riesgo cardiovascular. Un ritmo circadiano anómalo de la presión arterial podría aumentar el riesgo cardiovascular.

Objetivo: Determinar la existencia de una relación clínicamente relevante entre un ritmo circadiano anómalo de la presión arterial y un incremento del riesgo cardiovascular en pacientes con hipertensión arterial o hipertensión de bata blanca.

Material y Método: Estudio descriptivo en 166 pacientes mayores de 18 años del Área Básica de Salud de Balaguer que tuvieran una monitorización ambulatoria de la presión arterial realizada entre junio de 2014 y marzo de 2018 y cumplieran los criterios de inclusión y exclusión. Se realizó un análisis univariado y bivariado de las variables.

Resultados: Se obtuvo que en casi todos los promedios de presión arterial nocturna destaca el patrón riser mostrando que el $25 \%$ tenía un riesgo cardiovascular mayor o igual al $10 \%$, seguido del dipper extremo $16,67 \%$, dipper $9,4 \%$ y no dipper $8,27 \%$.

Conclusión: Un ritmo circadiano alterado se relaciona con un riesgo cardiovascular más elevado y un peor control de los factores que conllevan al mismo.

PALABRAS CLAVE: hipertensión; presión arterial;ritmo circadiano; enfermedades cardiovasculares, monitorización ambulatoria de la presión arterial.

Correspondencia:

Cristina García Serrano

Passatge Àngel Guimerà, 22. 25600 Balaguer. Lleida

E-mail: cristinagarse3@gmail.com

\section{Circadian rhytm of blood pressure and the rela- tion to cardiovascular risk factors}

\section{Abstract}

Introduction: Numerous studies have established both arterial hypertension and white coat hypertension as cardiovascular risk factors. An abnormal circadian rhythm of blood pressure may increase cardiovascular risk.

Objective: To determine the existence of a clinically relevant relationship between an abnormal circadian rhythm of blood pressure and an increase in cardiovascular risk in patients with hypertension or white coat hypertension.

Methods: A descriptive study in 166 patients older than 18 years was carried out. Patients were from the Basic Health Area of Balaguer, who had an ambulatory blood pressure monitoring, conducted between June 2014 and March 2018 and met the inclusion and exclusion criteria. Univariate and bivariate analyses of the variables were performed.

Results: In almost all means nighttime blood pressure, the riser pattern stands out, showing that $25 \%$ had a cardiovascular risk greater than or equal to $10 \%$, followed by extreme dippers $16.67 \%$, dippers $9.4 \%$ and non-dippers $8.27 \%$.

Conclusion: An altered circadian rhythm is related to a higher cardiovascular risk and a worse control of the related factors.

KEYWORDS: hypertension; blood pressure, circadian rhythm; cardiovascular diseases, ambulatory blood pressure monitoring. 


\section{Introducción}

La hipertensión arterial (HTA) ha sido claramente establecida como un factor de riesgo que contribuye a la progresión de aterosclerosis y enfermedades cardiovasculares. Del mismo modo, la hipertensión de bata blanca (HBB) definida como la HTA registrada únicamente en la consulta con valores de Monitorización Ambulatoria de la Presión Arterial (MAPA) dentro de la normalidad, también podría tener un impacto en el riesgo cardiovascular $(\mathrm{RCV})^{1}$. Varias investigaciones demuestran que la HBB podría ser un precursor de la HTA sostenida ${ }^{2}$. Además, diversos estudios defienden que la HBB se encuentra en un estado intermedio entre normotensión e HTA en cuanto a RCV, de ahí la importancia de realizar un seguimiento de la misma ${ }^{3}$.

El ritmo circadiano de la presión arterial (PA) se evalúa mediante la MAPA y da lugar a cuatro tipos de ritmo circadiano $0^{4,5}$. El perfil circadiano normal se caracteriza por el descenso de entre un $10 \%$ y un $20 \%$ de las cifras de PA nocturna frente a las cifras de PA diurna o de actividad (patrón dipper). La ausencia del descenso de las cifras de PA nocturna entre el $0 \%$ y el $10 \%$ se considera un patrón no dipper. Si el descenso de PA nocturna es mayor del $20 \%$ de las cifras diurnas se considera un patrón dipper extremo. Por el contrario, si la media de los valores de PA nocturna supera la media de la PA diurna se habla de patrón riser'.

Otra manera de definir el ritmo circadiano de la PA es mediante el cociente noche/día, de forma que los pacientes dipper presentarían un cociente entre 0,80 y 0,90 , los no dipper entre 0,91 y 1,00, los dipper extremo inferior a 0,80 y los risers mayor de $1,00^{6}$.

Estudios previos sugieren que un perfil circadiano anormal de la PA está asociado con un aumento del RCV en pacientes que ya han tenido un infarto de miocardio de etiología isquémica ${ }^{7}$. En España, se han realizado algunos estudios que así lo demuestran ${ }^{8}$. Sin embargo, esta relación no ha podido ser demostrada en pacientes que aún no han sufrido un debut de eventos cardiovasculares (ECV) ${ }^{4}$.

Por otro lado, sí se ha demostrado la relación entre el aumento de factores de riesgo cardiovascular (FRCV) (hipercolesterolemia, dislipemia, Índice de Masa Corporal (IMC) elevado y alteraciones de glucemia basal) en pacientes no depresores (no descenso de PA durante la noche con respecto al día)?

Según la guía de la American College of Cardiology/American Heart Association para la prevención, detección, evaluación y manejo de la hipertensión, muchos adultos con HTA tienen otros FRCV ${ }^{10}$. En un estudio de EEUU en adultos con HTA se observó que el $49,5 \%$ eran obesos, el $63,2 \%$ tenían hipercolesterolemia, el 27,2\% padecían diabetes mellitus (DM) y el $15,8 \%$ presentaban enfermedad renal crónica. Pero no sólo los FRCV son comunes entre los pacientes hipertensos, sino que pacientes diagnosticados con otros factores de riesgo, fueron diagnosticados de HTA. Por ejemplo, el $71 \%$ de los adultos estadounidenses diagnosticados de DM presentaban HTA ${ }^{11}$.

Por tanto, los FRCV modificables son comunes entre adultos con HTA incluyendo tabaquismo, DM, dislipemia, sobrepeso u obesidad, sedentarismo y/o dieta poco saludable.

Se plantea este estudio para describir si un ritmo circadiano anormal de la PA influye en una mayor prevalencia de otros FRCV y, por consiguiente, en un RCV más elevado que en aquellos que presentan un ritmo circadiano normal.

De esta manera, como objetivo principal del estudio nos hemos planteado determinar la existencia de una relación clínicamente relevante entre un ritmo circadiano anómalo de la PA, y un incremento del RCV en pacientes con HTA o HBB.

\section{Material y Método}

Se realizó un estudio descriptivo transversal en el ámbito de Atención Primaria del Área Básica de Salud (ABS) de Balaguer.

Como población de estudio se seleccionaron pacientes mayores de 18 años pertenecientes al ABS de Balaguer que tuvieran una MAPA realizada entre junio de 2014 y marzo de 2018. La población total fue de 173 pacientes. La muestra se obtuvo a través de una base de datos que contenía todos los registros de las MAPAs realizadas a los pacientes del ABS. El estudio consistió en la explotación de esa base de datos.

Los criterios de inclusión fueron hombres y mujeres, edad superior a 18 años, con diagnóstico de HTA (código diagnóstico CIE-10:I10), o bien diagnóstico de HBB (código diagnóstico CIE-10:R03.0). Como criterios de exclusión se establecieron: historia clínica inaccesible por cambio de ABS o exitus. Tras aplicar dichos criterios se obtuvo una muestra de 166 individuos con una exclusión de 7 pacientes. 
Los instrumentos utilizados fueron los registros de las MAPAs a través del monitor Spacelabs Healthcare modelo 90207-5Q y el cálculo de RCV por la escala Registre Gironí del Cor (REGICOR) mediante el programa informático de Atención Primaria ECAP.

La variable seleccionada como dependiente fue el patrón circadiano de la Presión Arterial Sistólica (PAS) y se clasificó en: dipper, dipper extremo, no dipper y riser.

Como variables independientes se analizaron: sexo (hombre, mujer), edad (entre 18 y 45 años, entre 45 y 65 años, entre 65 y 75 años, mayores de 75 años), tabaquismo (no fumador, fumador activo, ex-fumador), IMC (menor de 25 $\mathrm{kg} / \mathrm{m}^{2}$, entre 25 y $29 \mathrm{~kg} / \mathrm{m}^{2}$, superior o igual a $30 \mathrm{~kg} / \mathrm{m}^{2}$ ), diagnóstico DM (sí, no), colesterol total teniendo en cuenta que las categorías se diferenciaron entre DM y no DM por tener diferentes objetivos de control (pacientes con DM: inferior a $200 \mathrm{mg} / \mathrm{dl}$, superior o igual a $200 \mathrm{mg} / \mathrm{dl}$; pacientes sin DM: inferior a $250 \mathrm{mg} / \mathrm{dl}$, superior o igual a $250 \mathrm{mg} / \mathrm{dl}$ ), HDL (inferior a $45 \mathrm{mg} / \mathrm{dl}$, superior o igual a 45 $\mathrm{mg} / \mathrm{dl}$ ), triglicéridos (TG) (inferior a $200 \mathrm{mg} / \mathrm{dl}$, superior o igual $200 \mathrm{mg} / \mathrm{dll}$ ), filtrado glomerular (FG) según fórmula CKD-EPI (inferior a $60 \mathrm{ml} / \mathrm{min}$, superior o igual a $60 \mathrm{ml} /$ min), ECV (cardiopatía y/o enfermedad cerebrovascular isquémica; sí, no), RCV (REGICOR) (inferior a 10\%, superior o igual a $10 \%$ ), promedio de PAS despierto, promedio de Presión Arterial Diastólica (PAD) despierto, promedio de PAS dormido, promedio de PAD dormido, promedio de PAS $24 \mathrm{~h}$ y promedio de PAD $24 \mathrm{~h}$.

La recogida de datos se realizó a través de la historia clínica de Atención Primaria con el programa informático ECAP y a través del informe específico de cada MAPA. El análisis descriptivo univariante consistió en calcular la distribución en proporciones expresada en forma de porcentajes de sexo y grupo de edad en cada tipo de patrón circadiano de PA: dipper, dipper extremo, no dipper y riser.
Las valores cuantitativos de las variables tabaquismo, IMC, colesterol total, HDL, TG, FG, existencia de enfermedad cardiovascular y RCV, se calcularon en diferentes categorías por variable y también, se calculó su distribución en porcentajes en base a los patrones de ritmo circadiano de PA.

En el análisis bivariado se compararon los intervalos de confianza del $95 \%$ de las medias de los valores de colesterol total, colesterol HDL, colesterol LDL, TG, FG, puntuación en el índice de REGICOR, PAS despierto, PAD despierto, PAS dormido, PAD dormido, PAS 24 horas $y$ PAD 24 horas para cada tipo de patrón circadiano de PA. Se consideró que existían diferencias significativas entre los valores de cada variable en función del tipo de patrón circadiano de PA cuando los intervalos de confianza eran suficientemente alejados como para no solaparse.

En el presente estudio se garantiza la confidencialidad y anonimato de los datos suministrados conforme lo dispuesto en el Reglamento (UE) 2016/679 del Parlamento Europeo y el Consejo de 27 de abril de Protección de Datos (RGPD) y la normativa nacional de aplicación. Además, cuenta con el permiso previo de la dirección del centro y ha sido aprobado por el Comité Ético de Investigación Clínica del Instituto de Investigación en Atención Primaria Jordi Gol.

\section{Resultados}

En cuanto a la variable sexo, no se observan diferencias notables, excepto en el patrón dipper extremo en el que prevalece el sexo femenino con un $66,67 \%$. Se observa una disminución del porcentaje de pacientes con edad superior o igual a 45 años hasta mayores de 75 años en los patrones dipper, dipper extremo y no dipper. Sin embargo, en el patrón riser no varía el porcentaje con la edad, es decir, se mantiene constante en el 33,33\% (Tabla 1).

Tabla 1. Relación entre ritmo circadiano y variables sociodemográficas.

\begin{tabular}{|c|c|c|c|c|c|c|c|c|c|c|c|c|c|}
\hline & \multicolumn{4}{|c|}{$\operatorname{Sexo}\left(H^{*}, M^{* *}\right)$} & \multicolumn{9}{|c|}{ Edad (años) } \\
\hline & $n$ & H & $\%$ & $M$ & $\%$ & $18-45$ & $\%$ & $45-65$ & $\%$ & $65-75$ & $\%$ & $\geq 75$ & $\%$ \\
\hline Dipper & 64 & 27 & 42,18 & 37 & 57,82 & 12 & 18,75 & 29 & 45,31 & 19 & 29,69 & 4 & 6,25 \\
\hline $\begin{array}{l}\text { Dipper } \\
\text { extremo }\end{array}$ & 6 & 2 & 33,33 & 4 & 66,67 & 0 & 0,00 & 3 & 50,00 & 1 & 16,67 & 2 & 33,3 \\
\hline No dipper & 84 & 41 & 48,81 & 42 & 51,19 & 15 & 17,86 & 29 & 34,52 & 25 & 29,76 & 15 & 23,4 \\
\hline Riser & 12 & 5 & 41,67 & 7 & 58,33 & 0 & 0,00 & 4 & 33,33 & 4 & 33,33 & 4 & 33,3 \\
\hline
\end{tabular}

${ }^{*}$ Hombre, ${ }^{* *}$ Mujer. 
En el tabaquismo no se distingue ninguna relación clara ya que en todos los patrones circadianos prevalecen los no fumadores (Tabla 2).

Tabla 2. Relación entre ritmo circadianos y tabaquismo.

\begin{tabular}{|c|c|c|c|c|c|c|}
\hline & \multicolumn{6}{|c|}{ Tabaquismo } \\
\hline & No fumador & $\%$ & Fumador Activo & $\%$ & Ex fumador & $\%$ \\
\hline Dipper & 43 & 67,19 & 13 & 20,31 & 7 & 10,93 \\
\hline Dipper Extremo & 5 & 83,33 & 1 & 16,67 & 0 & 0,00 \\
\hline No dipper & 61 & 72,62 & 10 & 11,9 & 12 & 14,28 \\
\hline Riser & 9 & 75,00 & 0 & 0,00 & 2 & 16,67 \\
\hline
\end{tabular}

En cuanto al IMC, se observa un predominio de patrones dipper y no dipper en situación de sobrepeso (IMC mayor o igual a $25 \mathrm{~kg} / \mathrm{m}^{2}$ e inferior a $30 \mathrm{~kg} / \mathrm{m}^{2}$ ). Por otra parte, los patrones dipper extremo y riser destacan porque el $50 \%$ de la muestra presenta obesidad (IMC mayor o igual a $30 \mathrm{~kg} / \mathrm{m}^{2}$ ).

La hipercolesterolemia, establecida a partir de un colesterol total superior o igual a $200 \mathrm{mg} / \mathrm{dl}$ (en pacientes con DM) nos indica que prevalece el patrón riser frente al resto de patrones con un 33,33\%. En cuanto a pacientes no diabéticos y tomando como objetivo un colesterol total mayor o igual a $250 \mathrm{mg} / \mathrm{dl}$, se obtiene que predomina el patrón dipper con un $17,19 \%$.

En la medición de HDL establecida por debajo de $45 \mathrm{mg} /$ dl sobresale el patrón dipper con un 23,43\%.

El $16.67 \%$ de los pacientes con patrón riser presenta un nivel de TG por encima de $200 \mathrm{mg} / \mathrm{dl}$, dominando sobre el resto de los patrones.

EI FG por debajo de $60 \mathrm{ml} / \mathrm{min}$ representa un 33,33\% de la muestra en el patrón dipper extremo, seguido del patrón riser con un $16,67 \%$ (Tabla 3).

al resto (Tabla 4). bla 5$)$.
El cálculo de índice REGICOR, situando el punto de corte en el $10 \%$ (RCV alto) permitió clasificar a los estudiados como no dipper $8,27 \%$, dipper $9,4 \%$, dipper extremo $16,67 \%$, y, finalmente, riser con el $25 \%$. Además, se detectaron porcentajes similares en los pacientes que habían presentado algún ECV en los últimos 10 años: dipper extremo $(16,67 \%)$, riser $(16,67 \%)$ y no dipper (17,86\%). Sin embargo, los pacientes con patrón dipper (10,93\%) presentaron un porcentaje inferior de ECV

Tabla 4. RCV según el ritmo circadiano.

\begin{tabular}{lcccc}
\hline & \multicolumn{2}{c}{ ECV (sí, no) } & \multicolumn{2}{c}{ RCV (\%) } \\
& Sí & $\%$ & $\geq 10$ & $\%$ \\
Dipper & 7 & 10,93 & 6 & 9,40 \\
Dipper Extremo & 1 & 16,67 & 1 & 16,67 \\
No dipper & 15 & 17,86 & 7 & 8,27 \\
Riser & 2 & 16,67 & 3 & 25,00 \\
\hline
\end{tabular}

En cuanto al análisis bivariado, se observa que el patrón riser tiene medias ajustadas (según un intervalo de confianza del $95 \%$ ) superiores al resto de patrones circadianos en TG $(146,53 \mathrm{mg} / \mathrm{ml})$, PAS despierto $(130,95$ $\mathrm{mmHg})$, PAS dormido $(133,10 \mathrm{mmHg})$, PAD dormido $(77,74 \mathrm{mmHg})$, PAS 24 horas $(131,71 \mathrm{mmHg})$, PAD 24 horas $(76,26 \mathrm{mmHg})$ e inferiores en $\mathrm{FG}(73,77 \mathrm{ml} /$ min). Además, destaca un mayor valor del índice REGICOR en el patrón riser, de $6,10 \%$ frente a puntuaciones REGICOR inferiores en los otros patrones (dipper 4,92\%, dipper extremo 4,84\%, no dipper 5,82\%) (Ta-

Tabla 3. Relación entre ritmo circadiano y FRCV.

\begin{tabular}{|c|c|c|c|c|c|c|c|c|c|c|c|c|c|c|}
\hline & \multicolumn{4}{|c|}{ IMC $\left(\mathrm{kg} / \mathrm{m}^{2}\right)$} & \multicolumn{4}{|c|}{ Colesterol total (mg/dl) } & \multicolumn{2}{|c|}{ HDL (mg/dl) } & \multicolumn{2}{|c|}{$\mathrm{TG}(\mathrm{mg} / \mathrm{dl})$} & \multicolumn{2}{|c|}{$F G\left(\mathrm{ml} / \mathrm{min} / \mathrm{m}^{2}\right)$} \\
\hline & $25-30$ & $\%$ & $\geq 30$ & $\%$ & $\geq 200 \mathrm{DM}$ & $\%$ & $\geq 250$ no DM & $\%$ & $<45$ & $\%$ & $\geq 200$ & $\%$ & $<60$ & $\%$ \\
\hline Dipper & 34 & 53,16 & 20 & 31,25 & 6 & 9,38 & 11 & 17,19 & 15 & 23,43 & 8 & 12,50 & 4 & 6,25 \\
\hline $\begin{array}{l}\text { Dipper } \\
\text { Extremo }\end{array}$ & 2 & 33,33 & 3 & 50,00 & 1 & 16,67 & 0 & 0,00 & 0 & 0,00 & 0 & 0,00 & 2 & 33,33 \\
\hline No dipper & 42 & 50,00 & 24 & 23,57 & 12 & 14,26 & 2 & 2,38 & 15 & 17,86 & 6 & 7,40 & 8 & 9,52 \\
\hline Riser & 4 & 33,33 & 6 & 50,00 & 4 & 33,33 & 0 & 0,00 & 1 & 8,33 & 2 & 16,67 & 2 & 16,67 \\
\hline
\end{tabular}


Tabla 5. Análisis bivariado según SPSS v21 del ritmo circadiano respecto a FRCV.

\begin{tabular}{|c|c|c|c|c|c|c|c|c|}
\hline & \multicolumn{4}{|c|}{ DIPPER } & \multicolumn{4}{|c|}{ DIPPER EXTREMO } \\
\hline & \multirow{2}{*}{ Media } & \multicolumn{2}{|c|}{$\begin{array}{c}\text { Intervalo de confianza } \\
95 \%\end{array}$} & \multirow{2}{*}{$\begin{array}{l}\text { Media } \\
\text { ajustada }\end{array}$} & \multirow{2}{*}{ Media } & \multicolumn{2}{|c|}{$\begin{array}{c}\text { Intervalo de confianza } \\
95 \%\end{array}$} & \multirow{2}{*}{$\begin{array}{c}\text { Media } \\
\text { ajustada }\end{array}$} \\
\hline & & $\begin{array}{l}\text { Margen } \\
\text { inferior }\end{array}$ & $\begin{array}{l}\text { Margen } \\
\text { superior }\end{array}$ & & & $\begin{array}{l}\text { Margen } \\
\text { inferior }\end{array}$ & $\begin{array}{l}\text { Margen } \\
\text { superior }\end{array}$ & \\
\hline Colesterol Total & 201,30 & 191,30 & 211,30 & 199,63 & 204,25 & 165,72 & 242,78 & 203,39 \\
\hline HDL & 56,36 & 52,18 & 60,55 & 56,15 & 48,92 & 43,35 & 54,49 & 49,13 \\
\hline LDL & 125,35 & 116,27 & 134,43 & 124,18 & 129,83 & 94,79 & 164,88 & 128,88 \\
\hline TG & 128,81 & 108,12 & 149,50 & 121,89 & 127,33 & 98,33 & 156,33 & 127,20 \\
\hline $\mathbf{F G}$ & 81,18 & 77,44 & 84,91 & 82,62 & 80,08 & 66,63 & 93,53 & 82,31 \\
\hline REGICOR & 5,06 & 4,15 & 5,98 & 4,92 & 5,08 & 2,69 & 7,47 & 4,84 \\
\hline PAS despierto & 129,49 & 126,51 & 132,47 & 129,35 & 130,42 & 124,15 & 136,69 & 130,19 \\
\hline PAD despierto & 76,77 & 74,18 & 79,35 & 76,70 & 78,33 & 73,44 & 83,23 & 79,09 \\
\hline PAS dormido & 117,32 & 114,38 & 120,26 & 117,04 & 108,50 & 104,19 & 112,81 & 108,28 \\
\hline PAD dormido & 65,36 & 63,32 & 67,40 & 65,29 & 59,75 & 55,29 & 64,21 & 60,11 \\
\hline PAS $24 \mathrm{~h}$ & 125,51 & 122,71 & 128,38 & 125,41 & 123,75 & 117,89 & 129,61 & 123,39 \\
\hline \multirow[t]{4}{*}{ PAD $24 \mathrm{~h}$} & 73,09 & 70,77 & 75,40 & 72,92 & 72,58 & 67,73 & 77,43 & 73,20 \\
\hline & \multicolumn{4}{|c|}{ NO DIPPER } & \multicolumn{4}{|c|}{ RISER } \\
\hline & \multirow{2}{*}{ Media } & \multicolumn{2}{|c|}{$\begin{array}{c}\text { Intervalo de confianza } \\
95 \%\end{array}$} & \multirow{2}{*}{$\begin{array}{l}\text { Media } \\
\text { ajustada }\end{array}$} & \multirow{2}{*}{ Media } & \multicolumn{2}{|c|}{$\begin{array}{c}\text { Intervalo de confianza } \\
95 \%\end{array}$} & \multirow{2}{*}{$\begin{array}{l}\text { Media } \\
\text { ajustada }\end{array}$} \\
\hline & & $\begin{array}{l}\text { Margen } \\
\text { inferior }\end{array}$ & $\begin{array}{l}\text { Margen } \\
\text { superior }\end{array}$ & & & $\begin{array}{l}\text { Margen } \\
\text { inferior }\end{array}$ & $\begin{array}{l}\text { Margen } \\
\text { superior }\end{array}$ & \\
\hline Colesterol Total & 195,47 & 180,15 & 210,78 & 196,68 & 188,57 & 144,36 & 232,79 & 186,41 \\
\hline HDL & 52,53 & 47,79 & 57,27 & 51,87 & 49,86 & 41,32 & 58,39 & 50,23 \\
\hline LDL & 119,78 & 107,27 & 131,88 & 120,46 & 109,23 & 71,40 & 147,06 & 108,13 \\
\hline TG & 149,06 & 114,64 & 183,48 & 136,66 & 147,43 & 75,43 & 219,43 & 146,53 \\
\hline FG & 75,94 & 68,93 & 82,96 & 78,37 & 72,50 & 51,37 & 93,62 & 73,77 \\
\hline REGICOR & 9,13 & 2,13 & 16,13 & 5,82 & 6,32 & 1,17 & 11,47 & 6,10 \\
\hline PAS despierto & 131,34 & 126,19 & 136,50 & 130,39 & 130,86 & 114,55 & 147,16 & 130,95 \\
\hline PAD despierto & 74,19 & 70,30 & 78,08 & 74,47 & 75,86 & 65,80 & 85,91 & 75,79 \\
\hline PAS dormido & 126,16 & 121,46 & 130,85 & 125,99 & 132,14 & 117,43 & 146,86 & 133,10 \\
\hline PAD dormido & 70,09 & 66,32 & 73,87 & 70,37 & 77,71 & 67,52 & 87,91 & 77,74 \\
\hline PAS 24 h & 129,69 & 124,75 & 134,62 & 128,95 & 131,29 & 116,03 & 146,55 & 131,71 \\
\hline PAD $24 \mathrm{~h}$ & 72,88 & 68,99 & 76,76 & 73,15 & 76,29 & 66,19 & 86,38 & 76,26 \\
\hline
\end{tabular}

\section{Discusión}

Este estudio evaluó la relación de una alteración en el ritmo circadiano de la PAS con un aumento del RCV, encontrando un predominio del mismo entre el $25 \%$ de los riser y el $16,67 \%$ de los dipper extremo. En casi todos los promedios, excepto el promedio de PAD en el periodo despierto, el patrón riser destaca por encima del resto, lo que es clínicamente relevante. Estos resultados concuerdan con la mayoría de estudios analizados cuyas conclusiones radican en que una alteración del ritmo circadiano está asociada con un aumento del RCV. Doménech et al. posicionan a la HTA nocturna como un elemento más a integrar en el cálculo de RCV en el paciente hipertens $0^{6}$.

Recientemente han aparecido numerosas evidencias que sugieren que la reducción del descenso de las cifras de PA nocturna se asocia con un incremento de las complicaciones cardiovasculares, como el realiza- 
do por Sousa F. et al. quienes afirman que un cambio de no dipper a un patrón dipper, mejora el pronóstico cardiovascular ${ }^{12}$. Entre los daños identificados con una relación positiva con el patrón no dipper, se encuentra la presencia de hipertrofia ventricular izquierda. Cuspidi et al. lo estudiaron meticulosamente ${ }^{13}$.

Otras evidencias de daño cardiovascular objetivamente registradas son las detectadas en el estudio de Oliveras et al. donde se encontraron PA nocturnas asociadas a la presencia de microalbuminuria, reafirmando el papel de la PAS nocturna como marcador predictor de lesión orgánica ${ }^{14}$. En nuestro estudio, se puede observar un porcentaje mayor en $\mathrm{FG}$ por debajo de $60 \mathrm{ml} / \mathrm{min}$ en los patrones dipper extremo y riser.

Así, se establece el papel predictor de la alteración en el ritmo circadiano de la PA como inductor de daño orgánico en diversos territorios: cardíaco, renal y también vascular ${ }^{15}$. Syrseloudis et al. concluyeron que incluso en pacientes nunca tratados de una HTA ligera (entre los que podrían encontrarse nuestros pacientes de HBB), el ritmo circadiano es relevante a la hora de predecir la lesión orgánica subclínica ${ }^{16}$. Todos estos estudios pretenden evaluar el valor predictivo de la PA nocturna mediante la MAPA. En la mayoría de ellos, como en el de Hansen et al. se concluye el potente valor predictivo de la PA nocturna que, incluso ajustada a los valores de 24 horas, la relación noche/día y el patrón dipper mantienen su significado pronóstico de ECV7.

También, se ha encontrado un aumento de Síndrome de Apnea-Hipopnea Obstructiva del Sueño (SAHOS) $\mathrm{y}$ un peor descanso nocturno en patrones no dipper y riser $^{17,18}$.

No obstante, existen estudios, menos abundantes en la bibliografía, que no han evidenciado esta asociación, especialmente si los pacientes en cuestión aún no han sufrido ningún ECV ${ }^{4}$.

En algunos estudios, se han iniciado propuestas que pueden influir en una mejora del RCV en un ritmo circadiano alterado. Entre éstas se encuentran la promoción del ejercicio físico ${ }^{19}$, dieta saludable ${ }^{20,21}$, promoción de higiene del sueño, detección y cribaje de SAHOS ${ }^{17,18}$, aumento de la adherencia al tratamiento y control y seguimiento de enfermedades crónicas que puedan suponer otro riesgo añadido al RCV.

Otra de las acciones encaminadas a la prevención secundaria en estos pacientes podría ser detectar posibles placas de ateroma en carótidas ya que se ha visto en el presente estudio que el patrón riser destaca en niveles de colesterol total y TG fuera de objetivo. Hoy en día se promueve la ampliación de competencias por parte de enfermería, entre las que se encuentran la realización de ecografías del territorio vascular de las carótidas, aorta o femorales ${ }^{22}$.

Enfermería también podría actuar sobre la planificación, el diseño y la adherencia a una dieta que podría reducir no solo otros FRCV que influyen en la HTA y su ritmo circadiano (DM o dislipemia), sino que tienen su efecto sobre la propia $\mathrm{PA}^{20,21}$. Tanto en población coreana como británica hay estudios que demuestran con una evidencia significativa la influencia de los alimentos en la PA. En el primer estudio, a un sector de la población coreana se les retiró de su dieta habitual el café y alimentos ricos en grasas y azúcares, apreciándose una mejora de su $\mathrm{HTA}^{23}$. En el segundo, se realizó una intervención dietética sobre un grupo de pacientes británicos hipertensos evidenciándose no sólo la reducción de su PA sino también la relación de ésta con el RCV ${ }^{24}$.

No obstante, las herramientas fundamentales para el control de la HTA precisan a su vez de un control y una adherencia estrictas, siendo esta una de las piezas claves en la intervención de enfermería. Varios estudios han demostrado que un programa de apoyo y continuidad dirigido favorece una mejor adherencia al tratamiento ${ }^{25-27}$. Otros estudios sugieren que hace falta intensificar los esfuerzos en este sentido pues se han hallado cambios clínicamente significativos en la HTA de poblaciones sobre las que se han realizado programas de motivación para la adherencia y el autocuida$\mathrm{do}^{25,28,29}$.

Como limitaciones al estudio se contempla un sesgo de selección. Al tratarse de un estudio transversal en el tiempo, sólo están incluidos todos aquellos pacientes que tenían una MAPA realizada. Por tanto, se descartaron aquellos pacientes que no presentaron los criterios oportunos bien por decisión del profesional sanitario o bien por la no aceptación del paciente para realizarla.

Un ritmo circadiano alterado se relaciona con un riesgo cardiovascular más elevado y un peor control de los factores que conllevan al mismo.

\section{Conflicto de intereses}

No se declaran conflictos de interés por los autores. 
Recibido: 07-01-19

Revisado: 18-02-19

Modificado: 03-04-19

Aceptado: 25-04-19

\section{Bibliografía}

1. Franklin SS, Thijs L, Asayama K, Li Y, Hansen TW, Boggia $\mathrm{J}$ et al. The Cardiovascular Risk of White-Coat Hypertension. J Am Coll Cardiol 2016;68 (19):2033-43.

2. Robles NR. Importancia clínica de la hipertensión de bata blanca. Nefrología 2004;24(1):17-8.

3. Abolbashari M. White Coat Hypertension and Cardiovascular Diseases: Innocent or Guilty. Current Cardiology Report 2018;20(4):25.

4. Watanabe A, Hirashiki N, Murohara Y, Watanabe N, Kano N, Mori $\mathrm{H}$ et al. Abnormal Circadian Blood Pressure Profile as a Prognostic Marker in Patients with Nonischemic Dilated Cardiomyopathy. Cardiology 2017;136:1-9.

5. De La Sierra A, Gorostidi M, Banegas JR, Segura J, De La Cruz JJ, Ruilope LM. Nocturnal hypertension or nondipping: Which is better associated with the cardiovascular risk profile? Am J Hypertens 2014; 27(5):680-7.

6. Doménech CM, Sobrino J. Hipertensión nocturna. Hipertensión y Riesgo Vascular 2011;28(4):143-8.

7. Hansen TW, Li Y, Boggia J, Thijs L, Richart T, Staessen JA. Predictive role of the nighttime blood pressure. Hypertension 2011;57(1):3-10.

8. Ayala $D E$, Domínguez $M$, Otero $A$, Ríos $M T$, Gomara SM, Moya $A$ et al. Morning surge and sleep-time blood pressure as prognostic markers of cardiovascular risk: the hygia project. J Hypertens 2015;33 (1).

9. Mediavilla JD, Fernández C, Arroyo A, Jiménez J. Estudio del patrón circadiano de la presión arterial en pacientes hipertensos. An Med Interna 2007; 24(2):61-6.

10. Whelton PK, Carey RM, Aronow WS, Casey DE, CoIlins KJ, Dennison Himmelfarb C et al. 2017 ACC/ AHA/AAPA/ABC/ACPM/AGS/APhA/ASH/ASPC/ NMA/PCNA Guideline for the Prevention, Detection, Evaluation, and Management of High Blood Pressure in Adults: A Report of the American College of Cardiology/American Heart Association Task Force on Clinical Practice Guidelines. J Am Coll Cardiol 2018;71:127-248.

11. Egan BM, Li J, Hutchison FN, Ferdinand KC. Hypertension in the United States, 1999 to 2012: progress toward Healthy People 2020 goals. Circulation 2014;130:1692-9.

12. Sousa F, Neves J, Ferreira R, Polonia J, Bastos JM. In hypertension the change from a non-dipper to a dipper pattern is associated with a better cardiovascular prognosis than the persistence within the non-dipper pattern. J Hypertens 2015;33(1).

13. Cuspidi C, Giudici V, Negri F, Sala C. Nocturnal nondipping and left ventricular hypertrophy in hypertension: An updated review. Expert Review of Cardiovascular Therapy 2010;8(6):781-92.

14. Oliveras A, Armario P, Martell-Clarós N, Ruilope LM, De La Sierra A. Urinary albumin excretion is associated with nocturnal systolic blood pressure in resistant hypertensives. Hypertension 2011;57 (3):556-60.

15. Guerrero L, Fernández L, Andúgar J, Casal MC, Campo C, Segura J et al. Prevalencia de Vasculopatía Periférica Subclínica evaluada mediante el índice tobillo/brazo en pacientes con hipertensión arterial. Enferm Nefrol 2003;6(3):209-10.

16. Syrseloudis D, Tsioufis C, Andrikou I, Mazaraki A, Thomopoulos C, Mihas $C$ et al. Association of nighttime hypertension with central arterial stiffness and urinary albumin excretion in dipper hypertensive subjects. Hypertens Res 2011;34(1):120-5.

17. Sekizuka H, Osada N AY. The Factors Affecting the Non-dipper Pattern in Japanese Patients with Severe Obstructive Sleep Apnea. Intern Med 2018;57 (11):1553-9. 
18. Mokhlesi B, Hagen EW, Finn LA, Hla KM, Carter JR, Peppard PE. Obstructive sleep apnoea during REM sleep and incident non-dipping of nocturnal blood pressure: A longitudinal analysis of the Wisconsin Sleep Cohort. Thorax 2015;70(11):1062-9.

19. Kucukdurmaz Z, Karavelioglu Y, Karapinar H, Gul I, Yilmaz A, Yarlioglues $M$, et al. Hypertensive response to exercise in dipper and non-dipper normotensive diabetics. Clin Exp Hypertens 2014;36(5):275-9.

20. Yuzbashian E, Asgari G. Adherence to low-sodium dietary approaches to stop hypertension-stail diet may decrease the risk of incident chronic kidney disease among high-risk patients, a secondary prevention in prospective cohort study. Nephrol Dial Transplant 2018;33(7):1159-68.

21. Song S, Kim J. Gender differences in the association between dietary pattern and the incidence of hypertension in middle-age and older adults. Nutrients 2018;10(2):252.

22. Coll B, Betriu A, Feinstein $S B$, Valdivielso JM, Zamorano JL, Fernández E. Papel de la ecografía carotídea en la reclasificación del riesgo cardiovascular de sujetos de riesgo bajo-intermedio. Rev Esp Cardiol 2013;66(12):929-34.

23. Arribas Cobo $P$, Fernández Fuentes $S$, Guimerá Ferrer-Sama MA, Herrera Martín E, Martínez Aranda MA. Los efectos de una intervención educativa sobre la ingesta de sal para disminuir la sobrehidratación y la hipertensión arterial en pacientes con hemodiálisis. Enferm Nefrol 2013;16(3):87-8.

24. Jones NRV, Forohui NG, Khaw KT, Whareham NJ, Monsivais P. Accordance to the dietary approaches to stop hypertension diet pattern and cardiovascular disease in a British, population-base cohort. Eur J Epidemiol 2018;357:1794.
25. Haidari A, Moeini N, Khosravi A. The impact of peer support programme of adherence to the treatment regimen in patients with hypertension. A randomized clinical trial study. Iranian Journal of Nursing Mildwifery Research 2017;22(6):427-30.

26. Abel WM, Joyner JS, Cornelius JB, Greer DB. Self-care management strategies use by black women who self-report consistent adherence to antihypertensive medication. Patient Preference and Adherence 2017;11:1401-12.

27. Dasí MJ, Rojo E. Continuidad de los cuidados de enfermería: necesidad de coordinación entre niveles. Enferm Nefrol 2000;3(2):27-33.

28. Olowe OA, Ross AJ. Knowledge, adherence and control among patients with hypertension attending a peri-urban primary health care clinic, KwaZulu-Natal. African Journal of Primary Health Care and Family Medicine 2017;9(1):1-7.

29. Agbor VN, Takah NF, Aminde LN. Prevalence and factors associated with medication adherence among patients with hypertension in Sub-Sahara Africa. Protocol for a systematic review and meta-analysis. BMJ 2018;8(3):1-6.

Este artículo se distribuye bajo una Licencia Creative Commons Atribución-NoComercial 4.0 Internacional. https://creativecommons.org/licenses/by-nc/4.0/

Open Access (c) () () 